\title{
Regenerated Cellulose Fiber and Film Immobilized with Lysozyme
}

\section{Jonathan $\mathrm{Y}$ Chen ${ }^{1 *}$, Liangfeng Sun ${ }^{1}$ and Vincent Edwards $\mathbf{J}^{2}$}

${ }^{1}$ School of Human Ecology, The University of Texas at Austin, Austin, TX 78712, USA

${ }^{2}$ Southern Regional Research Center, USDA-ARS, New Orleans, LA, 70124, USA

\begin{abstract}
The present work reports an initial engineering approach for fabricating lysozyme-bound regenerated cellulose fiber and film. Glycine-esterified cotton was dissolved in an ionic liquid solvent 1-Butyl-3-methylimidazolium Chloride (BMIMCl) in which lysozyme was activated and covalently attached to cotton cellulose through an enzymatic conjugation between its carboxyl groups and glycine cellulose's amino groups. The resulting solution was extruded for fiber/film formation in a water bath. After performing a bicinchoninic acid (BCA) protein assay, quantity of attached lysozyme to cellulose fiber/film was evaluated. The study exhibited that a synthesis of lysozyme conjugation on cellulose in BMIMCl could be completed in a control manor, resulting in a cellulose solution suitable for fiber/film production. It was also found that lysozyme could be successfully immobilized onto the cellulose fiber and film regenerated from solution spinning with a reasonable amount ranging from 197.6 to $343.7 \mu \mathrm{g} / \mathrm{mL} . \mathrm{mg}$.
\end{abstract}

Keywords: Lysozyme immobilization; Ionic liquid; Regenerated cellulose fiber; Regenerated cellulose film; Esterified cotton; Bioactivity

\section{Introduction}

Biobased, bioactive, and biodegradable fiber/film biomaterials are increasingly expanding diverse industrial applications such as functional garments, household and personal care textiles, medical textiles, military textiles, bioactive filters, and bio-sensing food packing materials. Major technical advancement in producing antimicrobial textile products has been achieved in fabric finishing and coating processes. For example, by applying some special finish or coating agents such as triclosan, quaternary silicones, or poly hexamethylene biguanide (PHMB), antibacterial fabrics can be produced commercially and economically. To improve the durability of antimicrobial performance for those textile materials subject to daily wearing and frequent laundering, recent research efforts have been made for producing antimicrobial fibers. Some of the methods employed include adding an antimicrobial agent into spinning dopes as a filler [1] grafting a biocidal group onto fiber polymer branches [2,3] and covalently binding an enzyme group onto fiber polymer branches $[4,5]$.

Commercial cellulose fiber, mostly represented by cotton and rayon, is widely used for making apparel and consuming textiles because of its softness, moisture absorbency, easy care, and competitive price. Cellulose fiber is also biocompatible and biodegradable, and we have reported approaches for making bioactive cellulose fiber by immobilizing hydrolytic enzymes [6]. Hydrolase enzymes are capable of degrading bacterial cell walls to ultimately kill microorganisms. When they are attached to cellulose polymer branches, the cellulose fiber may exhibit antimicrobial activity. Surface adsorption and covalent coupling have been investigated as major methods of enzyme immobilization on cellulose.

Substantial progress in adsorbing enzymes to cellulose surfaces has been reported. Xyloglucan, xyloglucan endotransglycosylase, and chemically modified xyloglucan oligosaccharides were used to produce aminated xyloglucan adsorbed to cellulose surfaces [7]. Arola and his co-workers conjugated alkaline phosphatase to nano fibrillated cellulose activated by amine, epoxy, and carboxylic acid [8]. A fusion protein containing cellulose-binding-domain (CBD) was found effective and stable when adsorbed to cellulose [9-15]. Research work on adsorbing lysozyme to regenerated cellulose was reported by Suen and his co-workers [16].
To enable the covalent attachment of enzymes to cellulose-a polymeric structure support, activation of cellulose became a key step. Several activation methods were suggested to address this technical approach, among which the use of cyanogen bromide for activating polysaccharide supports appeared to be a commonly used method in previous research [17-27].

This activation reagent converted targeted hydroxyl groups into reactive alkoxide, making cellulose nucleophilic. Additionally, triethylamine could be used together with this reagent to enhance reagent's electrophilicity. In another approach, an organic compound 4-fluorobenzenesulfonate (fosyl chloride) was used to activate cellulosic hydroxyls to covalently link enzymes to the cellulose fiber [28]. A similar method of synthesizing aminocellulose derivatives by esterification of tosylcellulose was reported [29-31]. These aminocellulose derivatives were capable of being covalently coupled with exidoreductase enzymes such as glucose oxidase, lactate oxidase, or peroxidase for biosensor applications. Research was also done on a synthesis of attaching enzymes such as urease and lysozyme terminated with amines and carboxylic acid to cellulose and carboxymethylated cellulose [32-34]. Continuous efforts in this direction led to recent progress in the immobilization of lysozyme on citric acid treated cellulose and aminosilanated cellulose [35]

All the methods of enzyme immobilization on cellulose reviewed above either used cellulose polymer or used cellulose fiber as precursors. Recent investigation has been reported on use of ionic liquids to form regenerated cellulose film entrapped with laccase [36]. Similar work was also done on fabrication of cellulose and aminecontaining polymer composite film using ionic liquid solvents. After activation, this composite film was capable of conjugating enzymes

*Corresponding author: Jonathan $Y$ Chen, School of Human Ecology, The University of Texas at Austin, Austin, TX 78712, USA, Tel: +1-512-471-0633; E-mail: jychen2@austin.utexas.edu

Received February 27, 2014; Accepted June 27, 2014; Published July 04, 2014

Citation: Chen JY, Sun L, Edwards VJ (2014) Regenerated Cellulose Fiber and Film Immobilized with Lysozyme. Bioceram Dev Appl 4: 078. doi: 10.4172/2090-5025.1000078

Copyright: $\odot 2014$ Chen JY, et al. This is an open-access article distributed under the terms of the Creative Commons Attribution License, which permits unrestricted use, distribution, and reproduction in any medium, provided the original author and source are credited. 
such as laccase or lipase B aided by a cyanoborohydride coupling agent [37]. This paper presents a study on engineering approaches for the synthesis of enzyme conjugation on cellulose in ionic liquid solvents and for direct regeneration of enzyme-coupled cellulose fiber and film biomaterials. A major purpose of this research is to investigate if the conjugate reaction between cellulose and enzymes could be managed in ionic liquid solvents, and if enzymatic activity could be retained after fiber/film spinning process. The applied approach for the production of regenerated cellulose fiber/film is scalable and has potential to enable an eco-friendly conversion of cellulose biomass from wood, plants, and agricultural residues into high-end biomaterials.

\section{Materials and Methods}

\section{Raw materials and approach}

Scoured and bleached cotton cellulose nonwoven was produced and esterified in the Cotton Textile Chemistry Lab at the USDA Southern Regional Research Center. Lysozyme from chicken egg white 47,000 Units/mg protein solids, reagent 1-cyclohexyl-3-(2-morpholinoethyl)carbodiimide-metho-p-touene sulfonate (CMCS), and ionic liquid 1Butyl-3-methylimidazolium Chloride (BMIMCl) with purity $\geq 95 \%$ were purchased from Sigma-Aldrich, Inc. Other chemicals were also commercially available and were used as they were. The approach for producing regenerated cellulose fiber/film immobilized with lysozyme is illustrated by Figure 1. Processing steps involved in this approach include cellulose esterification and grinding, lysozyme activation, cellulose-lysozyme conjugation and dissolving, and cellulose fiber/film spinning, drying, and winding.

\section{Cellulose esterification}

The method of esterifying cotton cellulose was described in details in Edwards' work (Edwards et al. 2000). In brief, the scoured and bleached cotton cellulose nonwoven was pretreated with trifuoroacetic acid (TFA) and methylene chloride (DCM), and was esterified using Fmoc-glycine, diisopropylcarbodiimide (DIC), hydoxybenzotriazole (HOBT), and (dimethylamino) pyridine (DMAP). The Fmocglycine esterified cellulose was acetylated by acetic anhydride, $\mathrm{N}$-methylimidazole (NMI), and dimethylformamide (DMF).

\section{Lysozyme activation}

The reagent CMCS was used for activation of lysozyme carboxyl groups in the ionic liquid solvent BMIMCl. BMIMCl in amount of $88.2 \mathrm{~g}$ was preheated to $85^{\circ} \mathrm{C}$ using a planetary mixer. Then, lysozyme $(1.29 \mathrm{~g})$ and CMCS $(2.71 \mathrm{~g})$ were added into BMIMCl for mixing 1 min. During the activation, $\mathrm{pH}$ value of $\mathrm{BMIMCl}$ was justified to 8.0 by adding 2 drops of trientylamine.

\section{Cellulose-lysozyme conjugation}

The glycine-esterified cotton nonwoven sample was ground and added into BMIMCl for dissolving. The mixing operation was run for $2 \mathrm{hrs}$ and $15 \mathrm{mins}$ at $95^{\circ} \mathrm{C}$ with vacuuming and a $40-\mathrm{Hz}$ mechanical agitation. Covalent reaction completed during the cellulose dissolution in $\mathrm{BMIMCl}$, resulting in an enzymatic conjugation between the carboxyl groups of lysozyme and amino groups in the glycine-esterified cellulose matrix) (Figure 2).

\section{Formation of lysozyme-attached regenerated cellulose fiber/} film

The lysozyme-attached cellulose solution was fed into a desk-top extruder (LE-075, CSI Inc.) for fiber/film spinning. Temperatures of rotor and spinning head were set to $85^{\circ} \mathrm{C}$ and $110^{\circ} \mathrm{C}$ respectively. Rotor speed of the extruder was controlled within $65-85 \%$ of its full speed capacity $260 \mathrm{rpm}$, namely 169-221 rpm. Using a dry-wet spinning method, the cellulose solution was injected into a spin bath with tap water, where regenerated cellulose fiber/film was precipitated. Subject to a drawing force supplied by a take up device (CSI-194T, CSI Inc.), the produced fiber/film was drawn through a drying tube and wound on a spool. The spool take-up speed was $80 \mathrm{~mm} / \mathrm{s}$, allowing $11 \mathrm{~s}$ of drying time when drying temperature was set within $70-90^{\circ} \mathrm{C}$.

\section{Confirmation of lysozyme immobilization}

A BCA protein assay was performed on the fiber and film samples to examine the presence of protein and to quantify the protein amount. There was no dilution in the test using $1.5 \mathrm{mg} / \mathrm{mL}$ resulting in about $0.3375 \mathrm{mg}(1.5 \mathrm{mg} / \mathrm{mL} \times 0.225 \mathrm{~mL})$ tested in each well. The regenerated cellulose fiber/film was also tested using an FT-IR instrument Thermo Nicolet Avatar 370 (Thermo Electron Corporation) to examine if the lysozyme was covalently attached to the glycine cellulose.

\section{Surface and crystallinity characterization}

The fiber and film surface structure was examined using a scanning electron microscope (SEM) Zeiss Supra 40 VP (Carl Zeiss NTS $\mathrm{GmbH}$ ). The fiber crystallinity was measured using an instrument of wide angle X-ray diffraction (WAXD) RAPID II (Rigaku Americas Corporation). The test sample is a fiber bundle with a distance of 127.4 $\mathrm{mm}$ to the WAXD detector. To remove background noise, a blank control (without sample) test was also performed.

\section{Results and Discussion}

\section{Sample description and surface structure}

Information of all experimental samples is listed in Table 1. UT-Fiber and UT-Film are pure cellulose fiber and film samples respectively. They were regenerated from wood pulp and used as control samples. The samples of ARS-1,2,3 are lysozyme-immobilized cellulose fiber regenerated from esterified cotton. Samples ARS- 4 and ARS-5 are lysozyme-immobilized cellulose film also regenerated from esterified cotton. Figure 3 is a surface image of the fiber ARS- 1 obtained from the SEM instrument. Diameter of the experimental fiber samples is in the range of 30-50 $\mu \mathrm{m}$. Figure 4 shows surface morphology of the film ARS-4. Its surface is pretty smooth with a cross-sectional thickness around $20 \mu \mathrm{m}$.

\section{Lysozyme presence}

The test results of the lysozyme-cellulose conjugates on the regenerated cellulose microfibers are listed in Table 2. It can be seen that the lysozyme was covalently attached to the cellulose fiber with reasonable quantities. The FTIR measurement also confirmed a successful immobilization of lysozyme onto the cellulose fiber. As shown in Figure 5, the curve of lysozyme-attached cellulose fiber, labeled "USDA fiber," revealed a new N-H bond absorption peak at the frequency around $3000 \mathrm{~cm}^{-1}$, and significantly enhanced absorption at $1250 \mathrm{~cm}^{-1}$ by C-O-C bond and at $800 \mathrm{~cm}^{-1}$ by C-H bond.

\section{Fiber crystallinity}

Figure 6 exhibits the WAXD patterns and curves of the pure regenerated cellulose fiber (UT-Fiber) and lysozyme-bound cellulose fiber (ARS-1) for a direct comparison of the fiber crystalline structure. To extract the fiber crystalline profile, the WAXD 2D images were converted into $1 \mathrm{D}$ profile ( $2 \theta$ vs. intensity) via using the Rigaku 2DP software, followed by a curve fitting to establish a model that 
Citation: Chen JY, Sun L, Edwards VJ (2014) Regenerated Cellulose Fiber and Film Immobilized with Lysozyme. Bioceram Dev Appl 4: 078. doi: 10.4172/2090-5025.1000078

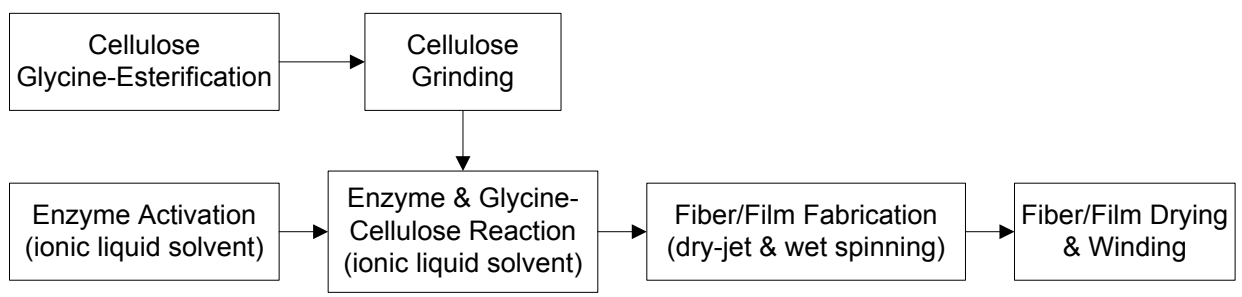

Figure 1: Process Flowchart

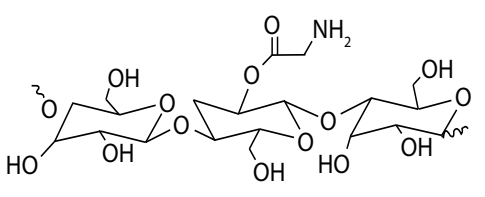

Glycine-Cellulose

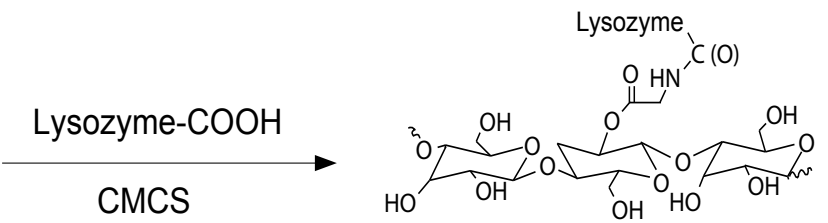

Bioactive Cellulose

Figure 2: Reaction scheme to immobilize lysozyme activated by CMCS.

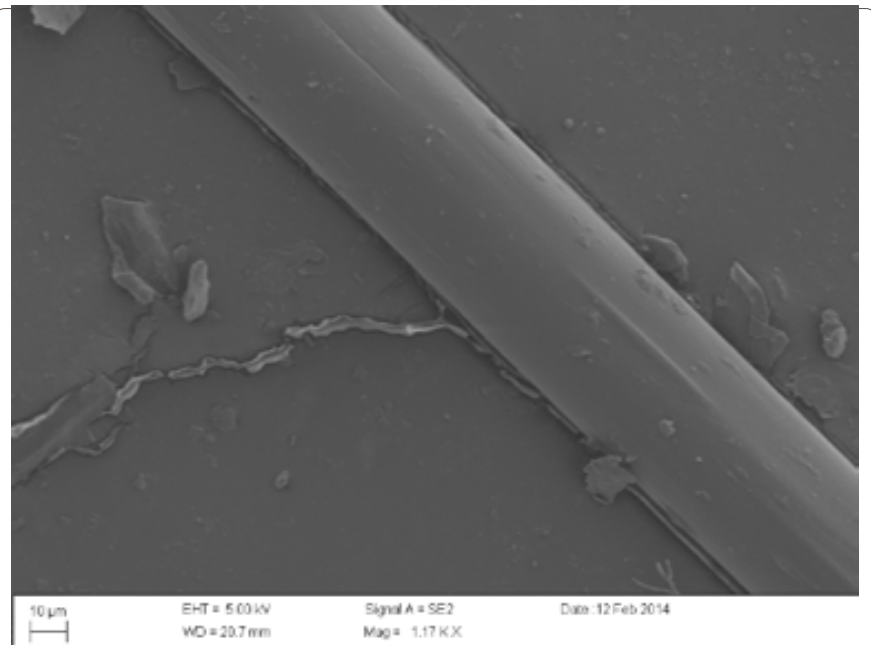

Figure 3: SEM of lysozyme-attached cellulose fiber (ARS-1 longitudinal view).



Figure 4: SEM of lysozyme-attached cellulose film (ARS-4)

\begin{tabular}{|c|l|c|c|}
\hline Sample ID & Description & Solution & Wash \& dry cycle \\
\hline UT-Fiber & Control fiber regenerated from wood & N/A & once \\
\hline UT-Film & Control film regenerated from wood & N/A & N/A \\
\hline ARS-1 & Fiber from unprotected glycine cotton & Part 1 & once \\
\hline ARS-2 & Fiber from unprotected glycine cotton & Part 2 & once \\
\hline ARS-3 & Fiber from unprotected glycine cotton & Part 2 & twice \\
\hline ARS-4 & Film from unprotected glycine cotton & Part 1 & N/A \\
\hline ARS-5 & Film from unprotected glycine cotton & Part 2 & N/A \\
\hline
\end{tabular}

Table 1: Experimental sample information

\begin{tabular}{|c|c|c|c|}
\hline Sample ID & Amt. in assay $(\mu \mathrm{g} / \mathrm{mL})$ & Actual amt. $(\mu \mathrm{g} / \mathrm{mL})$ & Protein* $(\mu \mathrm{g} / \mathrm{mL} . \mathrm{mg})$ \\
\hline UT-Fiber & 6.3 & - & - \\
\hline ARS-1 & 104.2 & 97.9 & 290.0 \\
\hline ARS-2 & 122.3 & 116 & 343.7 \\
\hline ARS-3 & 87.0 & 80.7 & 239.1 \\
\hline UT-Film & 12.4 & - & - \\
\hline ARS-4 & 79.1 & 66.7 & 197.6 \\
\hline ARS-5 & 98.2 & 85.8 & 254.2 \\
\hline
\end{tabular}

Table 2: Amount of lysozyme attached to regenerated cellulose

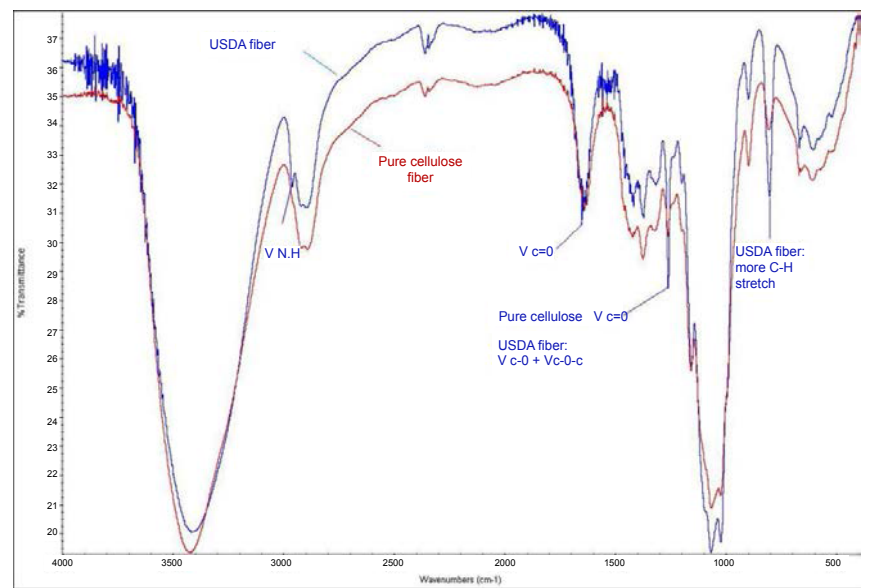

Figure 5: FTIR measurement: lysozyme-attached fiber ARS-1 (labeled "USDA fiber") vs. control sample UT-Fiber (labeled "Pure cellulose fiber"). 

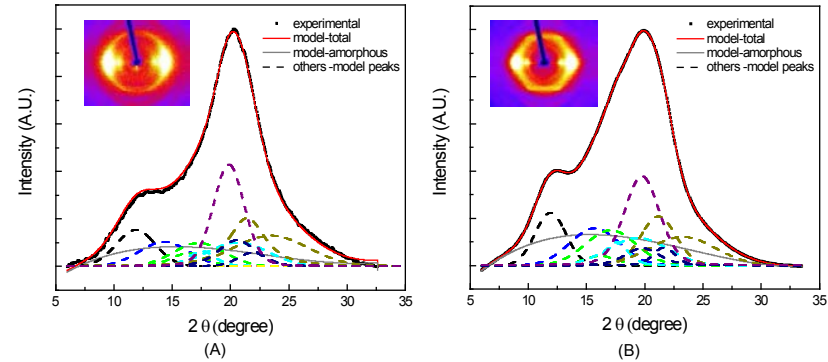

Figure 6: Fiber WAXD patterns and crystallinity profile: (A) UT-Fiber control sample; (B) ARS-1 sample.

enables separating the fiber crystalline and amorphous regions through peak deconvolution of the simulated WAXD curve (red line labeled "model-total"). From this analysis, it can be observed that the conjugation of lysozyme on cellulose chains affects the fiber crystalline structure. Overall, the lysozyme-bound cellulose fiber indicates a lower crystallinity $(79.28 \%)$ and bigger mean crystallite size $(2.33$ $\mathrm{nm})$, compared to the pure regenerated cellulose fiber with calculated crystallinity $(85.12 \%)$ and mean crystallite size $(2.14 \mathrm{~nm})$.

\section{Conclusions}

Lysozyme was activated and covalently attached to glycineesterified cotton cellulose dissolved in the BMIMCl ionic liquid solvent. Lysozyme-bound cellulose fiber and film was directly regenerated using the dry-wet spinning method. The BCA protein assay and FTIR measurement confirmed that lysozyme was immobilized onto the cellulose fiber and film with a reasonable amount ranging from 197.6 to $343.7 \mu \mathrm{g} / \mathrm{mL}$.mg. The developed method would enable coating protein on cellulose fiber/film products, rendering a possibility of producing diverse biomaterials where the protein is needed to place on fiber or film.

We conducted a turbidity assay for evaluating bioactivity of the lysozyme-attached cellulose fiber and film on M. lysodeikticus in 6-hr duration of activation. We found that there was no retention of activity for the lysozyme-attached cellulose fiber and film. Long cellulose dissolving time and high spinning temperature might be major reasons for the lysozyme inactivity. One way to solve this is to find a specific ionic liquid solvent that would be able to help reduce the cellulose dissolving time and lower the fiber/film spinning temperature.

\section{Acknowledgment}

The authors would like to thank Wei Jiang in the School of Human Ecology at The University of Texas at Austin for his assistance in the FTIR and WAXD measurements. Thanks are also extended to Yevgeniya S. Vinogradova in the Department of Biomedical Engineering at The University of Texas at Austin for helping the SEM and WAXD measurements.

\section{References}

1. El-Ola SMA (2008) Recent Developments in Finishing of Synthetic Fibers for Medical Applications. Designed Monomers and Polymers 11: 483-533.

2. Badrossamay MR, Sun G (2009) Durable and Rechargeable Biocidal Polypropylene Polymers and Fibers Prepared by Using Reactive Extrusion. J Biomed Mater Res B Appl Biomater 89: 93-101.

3. Sun YY, Sun G (2001) Novel regenerable N-halamine polymeric biocides. III. Grafting hydantoin-containing monomers onto synthetic fabrics. Journal of Applied Polymer Science 81: 517-525.

4. Edwards JV, Prevost N, Condon B, Sethumadhavan K, Ullah J, et al. (2011) Immobilization of Lysozyme on Cotton Fabrics: Synthesis, Characterization, and Activity. AATCC Review 11: 73-9
5. Nakamura M, Iwasaki T, Tokino S, Asaoka A, Yamakawa M, et al. (2011) Development of a Bioactive Fiber with immobilized Synthetic Peptides Designed from the Active Site of a Beetle Defensin. Biomacromolecules 12: 1540-1545.

6. Edwards JV, Sethumadhavan K, Ullah AHJ (2000) Conjugation and modeled structure/function analysis of lysozyme on glycine esterified cotton cellulosefibers. Bioconjugate Chemistry 11: 469-473.

7. Brumer H, Zhou Q, Baumann MJ, Carlsson K, Teeri TT (2004) Activation of crystalline cellulose surfaces through the chemoenzymatic modification of xyloglucan. J Am Chem Soc 126: 5715-5721.

8. Arola S, Tammelin T, Setala H, Tullila A, Linder MB (2012) ImmobilizationStabilization of Proteins on Nanofibrillated Cellulose Derivatives and Their Bioactive Film Formation. Biomacromolecules 13: 594-603.

9. Gilkes NR, Warren RAJ, Miller RC, Kilburn DG (1988) Precise Excision of the Cellulose Binding Domains from 2 Cellulomonas-Fimi Cellulases by A Homologous Protease and the Effect on Catalysis. J Biol Chem 263: 1040110407.

10. Levy I, Shoseyov O (2002) Cellulose-binding domains biotechnological applications. Biotechnology Advances 20: 191-213.

11. Ong E, Gilkes NR, Warren RAJ, Miller RC, Kilburn DG (1989) Enzyme Immobilization Using the Cellulose-Binding Domain of A Cellulomonas-Fimi Exoglucanase. BioTechnol 7: 604-607.

12. Ong E, Gilkes NR, Miller RC, Warren RAJ, Kilburn DG (1991) Enzyme Immobilization Using A Cellulose-Binding Domain - Properties of A BetaGlucosidase Fusion Protein. Enzyme Microb Technol 13: 59-65.

13. Ong E, Greenwood JM, Gilkes NR, Miller RC, Warren RAJ, et al. (1993) Proteins Designed for Adherence to Cellulose. Biocatalyst Design for Stability and Specificity 516: 185-194.

14. Owolabi JB, Beguin P, Kilburn DG, Miller RC, Warren RAJ (1988) Expression in Escherichia-Coli of the Cellulomonas-Fimi Structural Gene for Endoglucanase-B. Applied and Environmental Microbiology 54: 518-523.

15. Rincon MT, Cepeljnik T, Martin JC, Barak Y, Lamed R, et al. (2007) A novel cell surface-anchored cellulose-binding protein encoded by the sca gene cluster of Ruminococcus flavefaciens. Journal of Bacteriology 189: 4774-4783.

16. Suen SY, Lin SY, Chiu HC (2000) Effects of spacer arms on Cibacron Blue 3GA immobilization and lysozyme adsorption using regenerated cellulose membrane discs. Industrial \& Engineering Chemistry Research 39: 478-487.

17. Jin FX, Toda K (1988) Preparation of Immobilized Papain Covalently Bound on Natural Cellulose for Treatment of Beer. Biotechnology Letters 10: 221-223.

18. Jurado LA, Mosley J, Jarrett HW (2002 Sep 20) Cyanogen bromide activation and coupling of ligands to diol-containing silica for high-performance affinity chromatography: Optimization of conditions. J Chromatogr A 971: 95-104.

19. Kennedy JF, Paterson M (1993) Application of Cellulosic Fast-Flow Column Filters to Protein Immobilization and Recovery. Polymer Inter 32: 71-81.

20. Kohn J, Wilchek M (1981) Procedures for the Analysis of Cyanogen BromideActivated Sepharose Or Sephadex by Quantitative-Determination of Cyanate Esters and Imidocarbonates. Analytical Biochemistry 115: 375-382.

21. Kohn J, Wilchek M (1982) A New Approach (Cyano-Transfer) for CyanogenBromide Activation of Sepharose at Neutral Ph, Which Yields Activated Resins, Free of Interfering Nitrogen Derivatives. Biochemical and Biophysical Research Communications 107: 878-884.

22. Kohn J, Wilchek M (1983) 1-Cyano-4-Dimethylamino Pyridinium Tetrafluoroborate As A Cyanylating Agent for the Covalent Attachment of Ligand to Polysaccharide Resins. Febs Letters 154: 209-210.

23. Kohn J, Wilchek M (1984) The Use of Cyanogen-Bromide and Other Nove Cyanylating Agents for the Activation of Polysaccharide Resins. Applied Biochemistry and Biotechnology 9: 285-305.

24. March SC, Parikh I, Cuatreca P (1974) Simplified Method for CyanogenBromide Activation of Agarose for Affinity Chromatography. Analytical Biochemistry 60: 149-152.

25. Pasternak GW, March SC, Parikh I, Snyder SH, Cuatrecasas P (1976) Macromolecular Naloxone - Novel Long-Acting Polymer-Bound Drug. Life Sciences 18: 977-982. 
Citation: Chen JY, Sun L, Edwards VJ (2014) Regenerated Cellulose Fiber and Film Immobilized with Lysozyme. Bioceram Dev Appl 4: 078. doi: $10.4172 / 2090-5025.1000078$

Page 5 of 5

26. Tomar M, Prabhu KA (1985) Immobilization of Glucoamylase on Deae-Cellulose Activated with Chloride Compounds. Enzyme and Microbial Technology 7: 557-560.

27. Wilchek M, Miron T, Kohn J (1984) New Methods for Activation of Polysaccharides for Protein Immobilization and Affinity-Chromatography. Annals of the New York Academy of Sciences 434: 254-256.

28. Chang YA, Gee A, Smith A, Lake W (1992) Activating Hydroxyl-Groups of Polymeric Carriers Using 4-Fluorobenzenesulfonyl Chloride. Bioconjugate Chem 3: 200-202.

29. Berlin P, Klemm D, Tiller J, Rieseler R (2000) A novel soluble aminocellulose derivative type: its transparent film-forming properties and its efficient coupling with enzyme proteins for biosensors. Macromolecular Chemistry and Physics 201: 2070-2082.

30. Tiller J, Berlin P, Klemm D (2000) Novel matrices for biosensor applications by structural design of redox-chromogenic aminocellulose esters. Journal of Applied Polymer Science 75: 904-915.

31. Tiller J, Berlin P, Klemm D (1999 Jan 1) Soluble and film-forming cellulose derivatives with redox- chromogenic and enzyme immobilizing 1,4-phenylenediamine groups. Macromolecular Chemistry and Physics, 200: 1-9.
32. Dumitriu S, Popa M, Artenie V, Dan F (1989) Bioactive Polymers .56:Urease Immobilization on Carboxymethylcellulose, Biotechnol Bioeng 34: 283-290.

33. Edwards JV, Batiste SL, Gibbins EM, Goheen SC (1999) Synthesis and activity of $\mathrm{NH} 2$ - and $\mathrm{COOH}$-terminal elastase recognition sequences on cotton. J Pept Res 54: 536-543.

34. Yeon $\mathrm{KH}$, Lueptow RM (2006) Urease immobilization on an ion-exchange textile for urea hydrolysis. J Chem Technol Biotechnol 81: 940-950.

35. Edwards JV, Prevost N, Condon B, Sethumadhavan K, Ullah J, et al. (2011) Immobilization of Lysozyme on Cotton Fabrics: Synthesis, Characterization, and Activity. AATCC Review 11: 73-79.

36. Turner MB, Spear SK, Holbrey JD, Rogers RD (2004) Production of bioactive cellulose films reconstituted from ionic liquids. Biomacromolecules 5: 1379-1384.

37. Turner MB, Spear SK, Holbrey JD, Daly DT, Rogers RD (2005) Ionic liquidreconstituted cellulose composites as solid support matrices for biocatalyst immobilization. Biomacromolecules 6: 2497-2502. 